Novel differential algorithm to evaluate all the zeros of any generic polynomial

Francesco Calogero

To cite this article: Francesco Calogero (2017) Novel differential algorithm to evaluate all the zeros of any generic polynomial, Journal of Nonlinear Mathematical Physics 24:4, 469-472, DOI: https://doi.org/10.1080/14029251.2017.1375685

To link to this article: https://doi.org/10.1080/14029251.2017.1375685

Published online: 04 January 2021 
LETTER TO THE EDITOR

\title{
Novel differential algorithm to evaluate all the zeros of any generic polynomial
}

\author{
Francesco Calogero \\ Physics Department, University of Rome "La Sapienza", Italy \\ Istituto Nazionale di Fisica Nucleare, Sezione di Roma, Italy \\ francesco.calogero@roma1.infn.it,francesco.calogero@uniroma1.it
}

Received 28 June 2017

Accepted 16 August 2017

\begin{abstract}
A novel, remarkably neat, differential algorithm is introduced, which is suitable to evaluate all the zeros of a generic polynomial of arbitrary degree $N$.
\end{abstract}

\section{Introduction}

Recently a new differential algorithm to compute all the zeros of a generic polynomial has been introduced: it involves the solution of an appropriate system of $N$ nonlinearly coupled first-order Ordinary Differential Equations (ODEs) [1]. The novel technique described below is analogous, but it instead involves the solution of an appropriate system of $N$ nonlinearly coupled second-order ODEs. While this fact might be considered a drawback of the novel system when compared to the previous one [1], the novel technique introduced herein is remarkably neater and therefore worthy of being widely known at least because of its elegance.

\section{The task}

Let a generic (monic) polynomial be defined as follows in terms of its $N$ coefficients $c_{m}$ and its $N$ zeros $x_{n}$ :

$$
p_{N}(z)=z^{N}+\sum_{m=1}^{N}\left(c_{m} z^{N-m}\right)=\prod_{n=1}^{N}\left(z-x_{n}\right) .
$$

Hereafter we assume that $N$ is an arbitrary positive integer $N$, and that the $N$ coefficients $c_{m}$ are $N$, arbitrarily assigned, generally complex, numbers. The task is to compute the $N$ zeros $x_{n}$ (all different among themselves: "genericity" of the polynomial (2.1)). As it is well known this task can be generally performed explicitly only for $N=2, N=3$ respectively $N=4$, via equations involving square, cubic, respectively quartic roots. The $N$ coefficients $c_{m}$ can instead be expressed in an explicit manner in terms of the $N$ zeros $x_{n}: c_{m}=(-1)^{n} \sigma_{m}(\tilde{x})$, where $\sigma_{m}(\tilde{x})$ is the symmetrical sum of order $m$ of the $N$ zeros $x_{n}$ which are the $N$ elements of the unordered set $\tilde{x}$; but, remarkably, these formulas play no role in the following.

Hereafter indices such as $n, m, \ell$ run over the positive integers from 1 to $N$, unless otherwise indicated. 


\section{The algorithm}

Consider the dynamical system characterized by the following $N$ second-order ODEs (of Newtonian type: "accelerations equal forces")

$$
\ddot{\xi}_{n}(t)=\sum_{\ell=1 ; \ell \neq n}^{N}\left[\frac{2 \dot{\xi}_{n}(t) \dot{\xi}_{\ell}(t)}{\xi_{n}(t)-\xi_{\ell}(t)}\right],
$$

which characterizes the motion in the complex $\xi$-plane of the $N$ points identified by the $N$ coordinates $\xi_{n}(t)$; here and below superimposed dots indicate of course differentiation with respect to the real parameter $t$ ("dimensionless time": the independent variable). This system (3.1) is, in fact, an integrable Hamiltonian system, to which the honorary name "goldfish" has been attributed because of the neatness of its equations of motion, which are clearly autonomous and invariant under any arbitrary common translation or rescaling of the dependent variables $\xi_{n}(t)$ [2].

Next, integrate this system (3.1) of $N$ second-order ODEs from $t=0$ to $t=1$, starting-at the "initial time" $t=0$-from $N$, arbitrarily assigned, generally complex, $N$ initial values $\xi_{n}(0)$, with the corresponding initial velocities $\dot{\xi}_{n}(0)$ given by the following explicit formula in terms of these $N$ initial data $\xi_{\ell}(0)$ and of the $N$ coefficients $c_{m}$ of the polynomial (2.1):

$$
\dot{\xi}_{n}(0)=-\frac{\left[\xi_{n}(0)\right]^{N}+\sum_{m=1}^{N}\left\{c_{m}\left[\xi_{n}(0)\right]^{N-m}\right\}}{\prod_{\ell=1, \ell \neq n}^{N}\left[\xi_{n}(0)-\xi_{\ell}(0)\right]}=-\frac{p_{N}\left[\xi_{n}(0)\right]}{\prod_{\ell=1, \ell \neq n}^{N}\left[\xi_{n}(0)-\xi_{\ell}(0)\right]} .
$$

There thus obtain the $N$ zeros $x_{n}$ of the polynomial (2.1):

$$
\xi_{n}(1)=x_{n}, \quad p_{N}\left(x_{n}\right)=0 .
$$

\section{The proof}

Let the time-dependent monic polynomial

$$
P_{N}(z ; t)=z^{N}+\sum_{m=1}^{N}\left[\gamma_{m}(t) z^{N-m}\right]=\prod_{n=1}^{N}\left[z-\xi_{n}(t)\right]
$$

be characterized by its $N$ time-dependent coefficients $\gamma_{m}(t)$ and by its $N$ zeros $\xi_{n}(t)$. It has been recently shown [3] that the first and second time-derivatives of these quantities are related by the following convenient identities (for notational simplicity we omit below to indicate explicitly the $t$-dependence of the various quantities, whenever this omission is unlikely to cause any misunderstanding):

$$
\begin{gathered}
\dot{\xi}_{n}=-\frac{\sum_{m}^{N}\left[\dot{\gamma}_{m}\left(\xi_{n}\right)^{N-m}\right]}{\prod_{\ell=1, \ell \neq n}^{N}\left(\xi_{n}-\xi_{\ell}\right)}, \\
\ddot{\xi}_{n}=\sum_{\ell=1 ; \ell \neq n}^{N}\left(\frac{2 \dot{\xi}_{n} \dot{\xi}_{\ell}}{\xi_{n}-\xi_{\ell}}\right)-\frac{\sum_{m}^{N}\left[\ddot{\gamma}_{m}\left(\xi_{n}\right)^{N-m}\right]}{\prod_{\ell=1, \ell \neq n}^{N}\left(\xi_{n}-\xi_{\ell}\right)} ;
\end{gathered}
$$

while it is plain, see (4.1), that there hold the $N$ identities

$$
\left(\xi_{n}\right)^{N}+\sum_{m=1}^{N}\left[\gamma_{m}\left(\xi_{n}\right)^{N-m}\right]=0 .
$$


Now make the assignment

$$
\gamma_{m}(t)=\gamma_{m}(0)+t\left[c_{m}-\gamma_{m}(0)\right],
$$

which is obviously valid at $t=0$ and it clearly implies

$$
\ddot{\gamma}_{m}(t)=0, \quad \dot{\gamma}_{m}(t)=c_{m}-\gamma_{m}(0), \quad \gamma_{m}(1)=c_{m} .
$$

Clearly the first of these 3 identities (4.4b) implies (3.1) via (4.2b); at $t=0$, the second of these 3 identities (4.4b), via (4.2a) together with (4.3), implies (3.2); and the third of these 3 identities (4.4b) - together with the definitions of the polynomials $p_{N}(z)$ and $P_{N}(z ; t)$ in terms of their coefficients, see (2.1) and (4.1)—implies $p_{N}(z)=P_{N}(z ; 1)$ hence the validity of (3.3).

Remark 4.1. It is plain that the solutions $\xi_{n}(t)$ of the system (3.1) with arbitrary initial data $\xi_{n}(0)$ and with the initial velocities (3.2) are finite for all (finite) time-hence in particular for $0 \leq t \leq 1-$ since they are the zeros of the polynomial $P_{N}(z ; t)$, see (4.1), with the coefficients $\gamma_{m}(t)$ given by the simple prescription (4.4a). The system of nonlinear ODEs (3.1) might however feature a singularity — due to vanishing of the denominator in the right-hand side of (3.1)—for $0 \leq t \leq 1$ if in that time interval two (or more) of the points $\xi_{n}(t)$ moving in the complex $\xi$-plane collide. But clearly the set of $N$ initial data $\xi_{n}(0)$ causing such collisions has a vanishing measure in the space of all sets of initial data as long as one is working with complex numbers (as above recommended); so any eventual collision can be avoided by a slight change of the - a priori arbitrary-assignment of the initial data $\xi_{n}(0)$. Moreover any eventual collision does not entail a blow-up of the solutions of (3.1) — see above—but merely, after the collision, a "loss of identity" of the colliding points; not very relevant for the evaluation of the zeros $x_{n}$ of the polynomial (2.1), which have themselves no specific identity being the $N$ elements of the unordered set $\tilde{x}$, see above. On the other hand a collision of two or more of the moving points $\xi_{n}(t)$ at $t=1$ would be unavoidable if the polynomial $p_{N}(z)$, see (2.1), features one or more multiple zeros. For this reason the treatment in this paper is restricted to generic polynomials which by definition do not feature multiple zeros. A modification of the algorithm introduced in this paper—and of the previous analogous algorithm (see [1]) - to make them suitable to deal with polynomials featuring multiple zeros is possible but it requires a separate treatment (for appropriate hints see [4]).

Remark 4.2. Let us re-emphasize the flexibility of the algorithm described above entailed by the arbitrariness in the assignment of the $N$ initial data $\xi_{n}(0)$. It is moreover plain that if any one of these $N$ a priori arbitrary initial data $\xi_{n}(0)$ were to coincide with one of the zeros $x_{n}$ of the polynomial $p_{N}(z)$, see (2.1)—say, $\xi_{1}(0)=x_{1}$-then $\dot{\xi}_{1}(0)=0$ would follow (see (3.2)), hence as well $\dot{\xi}_{1}(t)=0$ (see (3.1)), implying that the coordinate $\xi_{1}(t)$ would remain fixed throughout its time evolution from $t=0$ to $t=1$; and moreover its presence would have no effect at all on the movement of the other points $\xi_{n}(t)$ with $n \neq 1$ (as clearly implied by (3.1)).

Remark 4.3. The preceding Remark 4.2 and the general character of the algorithm described above suggest that the numerical efficiency of that algorithm is likely to be improved the closer the assignment of the initial data $\xi_{n}(0)$ are to the (a priori unknown) zeros $x_{n}$. This suggest the possible advantage of an iterated application of the algorithm, with the outcome of a computational cycle used as input data for the next computational cycle-possibly with an appropriate sequential increase of the precision of the numerical routine employed to integrate from $t=0$ to $t=1$ the system of $N$ ODEs (3.1). 
Remark 4.4. While the preceding Remark 4.3 provides some potentially useful hints relevant to the applicability of the algorithm described above to actually compute the $N$ zeros of a given polynomial, clearly a detailed comparison in the context of numerical analysis of the merits/demerits of this technique-when compared to other standard numerical techniques to compute all the zeros of a given polynomial-is a nontrivial endeavour which exceeds the scope of this Letter and indeed the competence of its author.

Remark 4.5. Any new method to evaluate the zeros of a given polynomial is likely to be fruitful independently from its numerical proficiency, as a tool to obtain interesting information over other properties of the zeros of a polynomial than their numerical values. But is the method provided above indeed new?

A cogent answer to this question is hardly possible, since it would require a detailed scrutiny of the world mathematical literature spanning at least 4 centuries. It seems however reasonable to conjecture that the method presented above is indeed new, for the following 3 reasons. (i) A search via the web and by word of mouth had yielded no contrary indication when a somewhat analogous technique was recently introduced [1]. (ii) This state of affairs has persisted after the publication of that technique, which was also advertised to be new [1]. (iii) The neatness and elegance of the algorithm presented above suggests that, had it been previously discovered, it is extremely unlikely that it might have been subsequently forgotten.

\section{Envoy}

The results reported above have been obtained during the PMNP2017 International Conference held in Gallipoli, Italy, 17-24 June, 2017. It is a pleasure to thank the organizersB. Konopelchenko, G. Landolfi, L. Martina, R. Vitolo - and all the participants for the very pleasant and intellectually stimulating atmosphere throughout that meeting.

\section{References}

[1] F. Calogero, "Nonlinear differential algorithm to compute all the zeros of a generic polynomial", J. Math. Phys. 57, 083508 (4 pages) (2016); http://dx.doi.org/10.1063/1.4960821). arXiv:1607.05081v1 [math.CA]; "Comment on "Nonlinear differential algorithm to compute all the zeros of a generic polynomial" [J. Math. Phys. 57, 083508 (2016)]", J. Math. Phys. 57, 104101 (4 pages) (2016).

[2] F. Calogero, "The "neatest" many-body problem amenable to exact treatments (a "goldfish"?)", Physica D 152-153, 78-84 (2001); Classical Many-Body Problems Amenable to Exact Treatments, Lecture Notes in Physics m66, Springer, Heidelberg, 2001 (750 pages); Isochronous Systems, Oxford University Press, Oxford, 2008 (250 pages; marginally updated paperback version, 2012).

[3] F. Calogero, "New solvable variants of the goldfish many-body problem", Studies Appl. Math. 137(1), 123-139 (2016); DOI: 10.1111/sapm.12096.

[4] O. Bihun and F. Calogero, "Time-dependent polynomials with a multiple root, and related new solvable systems of nonlinear evolution equations", (in preparation). 